\title{
Injected Power Fluctuations in 1D Dissipative Systems
}

\author{
Jean Farago ${ }^{1 *}$ and Estelle Pitard ${ }^{2 \dagger}$ \\ ${ }^{1}$ Institut Charles Sadron CNRS-UPR 22 \\ 6 rue Boussingault BP 40016 F-67083 Strasbourg Cedex, France. \\ ${ }^{2}$ Laboratoire des Verres, Colloides et Nanomatériaux (CNRS-UMR 5587), CC69, \\ Université Montpellier 2, 34095 Montpellier Cedex 5, France.
}

\begin{abstract}
Using fermionic techniques, we compute exactly the large deviation function (ldf) of the time-integrated injected power in several one-dimensional dissipative systems of classical spins. The dynamics are $T=0$ Glauber dynamics supplemented by an injection mechanism, which is taken as a Poissonian flipping of one particular spin. We discuss the physical content of the results, specifically the influence of the rate of the Poisson process on the properties of the ldf.
\end{abstract}

Keywords: Glauber dynamics, spin systems, large deviation functions, free fermions, stochastic systems, fluctuation theorem.

\section{Introduction}

Fluctuations in nonequilibrium systems have attracted interest of physicists and mathematicians in recent years, due to fortuitous conjunctions of experiments [1, 2], which aimed at measuring and understanding the fluctuations of global variables in hydrodynamic experiments, and theoretical works [3, 4, 5, having stated new relations for the entropy production in some classes of nonequilibrium systems (the fluctuation theorems). This simultaneity was actually the cause of some bold assertions surmising a wider application of the so-called fluctuation theorems to dissipative systems [6, 7], whereas these relations were nothing but a manifestation of the time-reversal symmetry of the bulk dynamics. These conjectures were enforced by the apparent validity of the fluctuation relations in experiments and simulations, which yields incidentally a characteristic energy sometimes daringly termed "nonequilibrium temperature". Despite the fact that it has been convincingly proven that this apparent validity is only due to our inability of probing the large deviation functions of injected power in zones of negative injected power [8, 9, 10, 11, 12, 13], it is however interesting to show explicit examples of dissipative systems where the large deviation function (ldf) of injected power can be exactly computed, all the more so there exists very few such exact calculations on dissipative systems

*e-mail: farago@ics.u-strasbg.fr

${ }^{\dagger}$ e-mail: estelle.pitard@lcvn.univ-montp2.fr 
[9, 10, 14, 15, 16, for which the absence of detailed balance condition makes the situation more difficult to handle.

Moreover, for conservative systems in nonequilibrium stationary states, interesting relations on the related ldf of integrated current have been discovered recently [17, and it is interesting to compare the classes of conservative and dissipative systems as regards the fluctuations of the global variable associated with the external excitation (the very cause of the nonequilibrium state).

In this paper, we consider a 1D system of $2 N(N \rightarrow \infty)$ classical spins on a ring, labelled from $-N$ to $N-1$, updated according a $T=0$ Glauber dynamics, and supplemented by an injection mechanism. The $T=0$ Glauber dynamics is defined as follows : the probability for a spin $s_{j}$ to flip between $t$ and $t+d t$ is given by $d t\left[1-s_{j}\left(s_{j-1}+s_{j+1}\right) / 2\right]$, that is, $s_{j}$ flips with a rate 1 if its neighbours are in different states, cannot flip if $s_{j}=s_{j-1}=s_{j+1}$ and flips with a rate 2 if $-s_{j}=s_{j-1}=s_{j+1}$. It is important to mention that these dynamics are dissipative: a flip either does not modify the energy of the system, or lessens it. As a result, energy must be injected by an additional mechanism of injection in order to drive the system into a non trivial stationary state. We will consider two different models of injection : for both models (labelled I and II hereafter), only the zeroth spin $s_{0}$ (the "external boundary" of the system) flips randomly as a Poisson process with a parameter $\lambda$. The difference between the two models lies in the ability of the zeroth spin, besides the Poissonian flip, to spontaneously flip under the Glauber rules (model II) or not (model I); it is qualitatively more important than it could appear, as in one case (model II) both halves of the system are dynamically connected in the vicinity of $s_{0}$ when in the other (model I) they are disconnected. In the thermodynamic limit, Model I can be viewed as two independent identical systems driven out of equilibrium by $s_{0}$.

For these systems, we computed exactly $f(p)$, the large deviation function of the injected energy, a major observable associated with the fluctuations of the energy flux in stationary systems. It is defined as

$$
f(p)=\lim _{\tau \rightarrow \infty} \tau^{-1} \log \operatorname{Prob}((\text { energy injected between } 0 \text { and } \tau) / \tau=p)
$$

As the dynamics are stochastic, no explicit formula can be put forward for $\Pi$ the energy injected in the system by $\lambda$ from $t=0$ to $t=\tau$, but fortunately such expressions are not needed for our purpose. In section 2, we expose the dynamics of the models in greater detail, as well as the computation and the exact results for $g(\alpha)$, the ldf of the characteristic function. In section 3 we numerically solve the inverse Legendre transforms and get the ldfs for the injected energy for the two models considered here. We discuss their physical properties, in particular their variations with respect to the change of the injection rate.

\section{Fermionic approach to the injected power}

It is useful to describe spin systems in the dual representation of the domain walls : between the site 0 and 1 is located the locus of a possible domain wall labelled 0 , and so forth. The state of the system is thus characterized by $\mathcal{C}=\left(n_{-N}, \ldots, n_{-1}, n_{0}, n_{1}, n_{2}, \ldots, n_{N-1}\right)$, where the $n_{i}$ are either 0 (no domain wall) or 1 . There is $2^{2 N}$ possible states in this representation, in contrast with the spin representation where a degeneracy $s \leftrightarrow-s$ doubles 
this number. The dynamical equation for the probability is given by

$$
\partial_{t} P(\mathcal{C})=\lambda\left[P\left(\mathcal{C}_{0}\right)-P(\mathcal{C})\right]+\sum_{j}\left[P\left(\mathcal{C}_{j}\right) w\left(\mathcal{C}_{j} \rightarrow \mathcal{C}\right)-P(\mathcal{C}) w\left(\mathcal{C} \rightarrow \mathcal{C}_{j}\right)\right]
$$

where $\mathcal{C}_{j}$ holds for the state $\mathcal{C}$ whose domain wall variables $n_{j}$ and $n_{j-1}$ have been changed (according to $n \rightarrow 1-n$ ). The $T=0$ Glauber dynamics corresponds to

$$
\begin{aligned}
& w\left(\mathcal{C}_{j} \rightarrow \mathcal{C}\right)=2-n_{j}-n_{j-1} \\
& w\left(\mathcal{C} \rightarrow \mathcal{C}_{j}\right)=n_{j}+n_{j-1}
\end{aligned}
$$

where $n_{j}$ and $n_{j-1}$ are the variables associated with the state $\mathcal{C}$ (we use this convention hereafter). Note that models I and II differ in the preceding equations in the way the summation over $j$ is carried out: either it is not restricted (model II), or index $j=0$ is implicitely removed (model I).

We consider that each domain wall contributes as a quantum of energy 1 to the global energy of the system. We are interested in the energy $\Pi$ injected into the system up to time $t$ by the poissonian injection. Following [18], the route to this time integrated observable begins with the consideration of the joint probability $P(\mathcal{C}, \Pi, t)$, the probability for the system to be in the state $\mathcal{C}$ at time $t$ having received the energy $\Pi$ from the injection. The dynamical equation for this quantity is readily

$$
\begin{aligned}
& \partial_{t} P(\mathcal{C}, \Pi)=\lambda\left\{P\left(\mathcal{C}_{0}, \Pi-2\right) n_{0} n_{-1}+P\left(\mathcal{C}_{0}, \Pi+2\right)\left(1-n_{0}\right)\left(1-n_{-1}\right)\right. \\
& \left.+P\left(\mathcal{C}_{0}, \Pi\right)\left[\left(1-n_{0}\right) n_{-1}+\left(1-n_{-1}\right) n_{0}\right]-P(\mathcal{C}, \Pi)\right\}+\sum_{j}\left[P\left(\mathcal{C}_{j}, \Pi\right) w\left(\mathcal{C}_{j} \rightarrow \mathcal{C}\right)-P(\mathcal{C}, \Pi) w\left(\mathcal{C} \rightarrow \mathcal{C}_{j}\right)\right]
\end{aligned}
$$

We define next the generating function of $\Pi$ as

$$
F(\mathcal{C})=\sum_{\Pi=-\infty}^{\infty} e^{\alpha \Pi} P(\mathcal{C}, \Pi)
$$

This quantity, summed up over the states, yields the generating function $\langle\exp (\alpha \Pi)\rangle$ from which one derives its ldf $g(\alpha)$ :

$$
\left\langle e^{\alpha \Pi}\right\rangle \underset{t \rightarrow \infty}{\simeq} e^{t g(\alpha)}
$$

This ldf $g(\alpha)$ is closely related to $f(p)$, the ldf of the probability density function of $\Pi$, as they are Legendre transform of each other [9] :

$$
\begin{aligned}
\operatorname{Prob}(\Pi / t=p) \underset{t \rightarrow \infty}{\propto} \exp (t f(p)) \\
f(p)=\min _{\alpha}(g(\alpha)-\alpha p)
\end{aligned}
$$

Let us write the dynamical equation for $F$ :

$$
\begin{aligned}
& \partial_{t} F(\mathcal{C})= \\
& \begin{aligned}
\lambda\left[e^{2 \alpha} F\left(\mathcal{C}_{0}\right) n_{0} n_{-1}+e^{-2 \alpha} F\left(\mathcal{C}_{0}\right)(1-\right. & \left.\left.n_{0}\right)\left(1-n_{-1}\right)+F\left(\mathcal{C}_{0}\right)\left[\left(1-n_{0}\right) n_{-1}+\left(1-n_{-1}\right) n_{0}\right]-F(\mathcal{C})\right] \\
& +\sum_{j}\left[F\left(\mathcal{C}_{j}\right)\left(2-n_{j}-n_{j-1}\right)-F(\mathcal{C})\left(n_{j}+n_{j-1}\right)\right]
\end{aligned}
\end{aligned}
$$


The function $g(\alpha)$ is closely related to the dynamical matrix at work in the rhs of the preceding equation, since it is in general its largest eigenvalue. Thus, succeeding in computing $g(\alpha)$ is not as complicated as knowing the whole dynamics (eigenvalues and eigenvectors), but nevertheless it remains a difficult challenge, as the whole spectrum of the matrix has to be known.

Fortunately, our problem belongs to the category of the "free-fermions" problems, for which a diagonalization of the dynamics into independent "modes" can be achieved. To do so, we construct the state vector as

$$
|\phi\rangle=\sum_{\mathcal{C}} F(\mathcal{C})|\mathcal{C}\rangle
$$

where $|\mathcal{C}\rangle$ is a vector in the $2^{2 N+1}$ dimensional tensorial product of the space of states of domain walls (each of dimension 2). The dynamical evolution of $|\phi\rangle$ yields expressions like $\sum_{\mathcal{C}} F\left(\mathcal{C}_{0}\right) n_{0} n_{-1}|\mathcal{C}\rangle$ which can be represented as

$$
\begin{aligned}
\sum_{\mathcal{C}} F\left(\mathcal{C}_{0}\right) n_{0} n_{-1}|\mathcal{C}\rangle & =\sum_{\mathcal{C}} F\left(\mathcal{C}_{0}\right) \widehat{n}_{0} \widehat{n}_{-1}|\mathcal{C}\rangle \\
& =\widehat{n}_{0} \widehat{n}_{-1} \sum_{\mathcal{C}} F(\mathcal{C})\left|\mathcal{C}_{0}\right\rangle \\
& =\widehat{n}_{0} \widehat{n}_{-1} \sigma_{0}^{x} \sigma_{-1}^{x}|\phi\rangle=s_{0}^{-} s_{-1}^{-}|\phi\rangle
\end{aligned}
$$

where

$$
\widehat{n}=\left(\begin{array}{ll}
0 & 0 \\
0 & 1
\end{array}\right), \quad \sigma^{x}=\left(\begin{array}{ll}
0 & 1 \\
1 & 0
\end{array}\right), \quad s^{-}=\left(\begin{array}{ll}
0 & 0 \\
1 & 0
\end{array}\right)
$$

(we will have use also of $s^{+}={ }^{t}\left(s^{-}\right)$; note that $s^{-} s^{+}=\widehat{n}$ ). Note that for the time being, operators with different particle indices commute. Similar computations on other terms allows to write the dynamical equation for $|\phi\rangle$ as

$$
\begin{aligned}
\partial_{t}|\phi\rangle= & H|\phi\rangle \text { where } \\
H= & \lambda\left[e^{2 \alpha} s_{0}^{-} s_{-1}^{-}+e^{-2 \alpha} s_{0}^{+} s_{-1}^{+}+s_{0}^{+} s_{-1}^{-}+s_{0}^{-} s_{-1}^{+}-1\right] \\
& +\sum_{j}\left[2 s_{j-1}^{+} s_{j}^{+}+s_{j-1}^{+} s_{j}^{-}+s_{j-1}^{-} s_{j}^{+}-s_{j}^{-} s_{j}^{+}-s_{j-1}^{-} s_{j-1}^{+}\right]
\end{aligned}
$$

The fermionization of this "Hamiltonian" proceeds using the Jordan-Wigner transformation

$$
\begin{aligned}
& c_{-N}=s_{\operatorname{def}}^{+} \\
& c_{-N}^{\dagger}=s_{-N}^{-} \\
& c_{j}=s_{j}^{+} \sigma_{-N}^{z} \sigma_{-N+1}^{z} \cdots \sigma_{j-1}^{z} \\
& c_{j \operatorname{def}}^{\dagger}=s_{j}^{-} \sigma_{-N}^{z} \sigma_{-N+1}^{z} \cdots \sigma_{j-1}^{z}
\end{aligned}
$$

where

$$
\sigma^{z}=\left(\begin{array}{rr}
1 & 0 \\
0 & -1
\end{array}\right)
$$


is the third Pauli matrix. A major change provided by this transformation is that now operators with different indices do no longer commute. Instead, it can be verified that for all $i$ and $j$

$$
\begin{aligned}
& \left\{c_{i}, c_{j}\right\}=0 \\
& \left\{c_{i}^{\dagger}, c_{j}^{\dagger}\right\}=0 \\
& \left\{c_{i}^{\dagger}, c_{j}\right\}=\delta_{i, j}
\end{aligned}
$$

where the bracket is defined by $\{a, b\}=a b+b a$. These relations, characteristic of fermionic operators, yields a useful rewriting of the dynamical operator

$$
H=\lambda\left[e^{2 \alpha} c_{-1}^{\dagger} c_{0}^{\dagger}+e^{-2 \alpha} c_{0} c_{-1}+c_{0}^{\dagger} c_{-1}+c_{-1}^{\dagger} c_{0}-1\right]+\sum_{j}\left[2 c_{j} c_{j-1}+c_{j}^{\dagger} c_{j-1}+c_{j-1}^{\dagger} c_{j}-c_{j}^{\dagger} c_{j}-c_{j-1}^{\dagger} c_{j-1}\right]
$$

The change of variables $\tilde{c}^{\dagger}=e^{\alpha} c^{\dagger}, \tilde{c}=e^{-\alpha} c$ is compatible with the fermionic structure and allows the rewriting of the preceding equation (we omit the tildas in the following) :

$H=\lambda\left[c_{-1}^{\dagger} c_{0}^{\dagger}+c_{0} c_{-1}+c_{0}^{\dagger} c_{-1}+c_{-1}^{\dagger} c_{0}-1\right]+\sum_{j}\left[2 e^{2 \alpha} c_{j} c_{j-1}+c_{j}^{\dagger} c_{j-1}+c_{j-1}^{\dagger} c_{j}-c_{j}^{\dagger} c_{j}-c_{j-1}^{\dagger} c_{j-1}\right]$

In order to achieve the diagonalization, we follow the route described in the Appendix. The Hamiltonian can be written as

$$
H=\sum_{q} \Lambda_{q}\left(\xi_{q}^{\dagger} \xi_{q}-\frac{1}{2}\right)-2 N+1-\varepsilon-\lambda
$$

where $\varepsilon$ is defined as:

$$
\varepsilon= \begin{cases}0 & (\text { model I }) \\ 1 & (\text { model II })\end{cases}
$$

The largest eigenvalue of $H$ is thus given by

$$
g(\alpha)=\frac{1}{2} \sum_{q}\left|\Lambda_{q}\right|-2 N+1-\varepsilon-\lambda
$$

provided all the $\Lambda_{q}$ are real. If one takes into account possible complex eigenvalues, $g(\alpha)$ will be rather defined by

$$
g(\alpha)=\frac{1}{2} \sum_{q}\left|\operatorname{Re}\left(\Lambda_{q}\right)\right|-2 N+1-\varepsilon-\lambda
$$

As explained in the appendix, $\left\{\Lambda_{q},-\Lambda_{q}\right\}_{q}$ is the spectrum of the matrix

$$
M_{0}=\left(\begin{array}{rr}
A & B \\
D & -A
\end{array}\right)
$$

with the notations of the appendix; moreover we can compute its characteristic polynomial from (93), taking advantage of $B$ almost empty. We define $E(\mu)=(A+\mu)^{-1} D(A-\mu)^{-1}$ and (93) shows that we have to compute $\operatorname{det}(1+B E)$; the matrix $(1+B E)$ is quite 
simple, since $B=\lambda\left(M_{-1,0}-M_{0,-1}\right)$, where $\left\{M_{i j}\right\}_{i j}$ is the canonical basis of the $2 N \times 2 N$ matrices. As a result $B E$ is zero except on the lines -1 and 0 , and one has

$$
\begin{aligned}
\operatorname{det}(1+B E) & =\left(1-\lambda E_{-1,0}\right)\left(1+\lambda E_{0,-1}\right)+\lambda^{2} E_{0,0} E_{-1,-1} \\
& =\left(1+\lambda E_{0,-1}(-\mu)\right)\left(1+\lambda E_{0,-1}(\mu)\right)+\lambda^{2} E_{0,0}(\mu) E_{-1,-1}(\mu)
\end{aligned}
$$

where we exploited the fact that $E^{T}(\mu)=-E(-\mu)$. Note in passing that the symmetry of this expression with respect to $\mu \rightarrow-\mu$, demonstrated with general arguments in the appendix, is blatant here, as the $E_{j j}$ are antisymmetric functions of $\mu$.

A further simplification is provided by the fact that the physical system has itself a symmetry $i \leftrightarrow-i-1$ (expressed in the matrices $A, B$ and $D$ as $X_{i, j}=X_{-j-1,-i-1}$ ) which gives $E_{00}(\mu)=-E_{-1,-1}(\mu)$ and $E_{0,-1}(\mu)=E_{0,-1}(-\mu)$, whence

$$
\operatorname{det}(1+B E)=\left[1+\lambda E_{0,-1}(\mu)+\lambda E_{0,0}(\mu)\right]\left[1+\lambda E_{0,-1}(\mu)-\lambda E_{0,0}(\mu)\right]
$$

The next step is to compute the elements $(0, j)$ and $(-1, j)$ of the matrix $(\mu \operatorname{Id}-A)^{-1}$, or equivalently the associated cofactors of $\mu \mathrm{Id}-A$, termed $\operatorname{Cof}(i, j)$. Precisely, we need the cofactors $\operatorname{Cof}(0, j)$, and the others are obtained using $\operatorname{Cof}(i, j)=\operatorname{Cof}(j, i)$ and $\operatorname{Cof}(i, j)=$ $\operatorname{Cof}(-i-1,-j-1)$. We get :

$$
\left[(\mu-A)^{-1}\right]_{0, j}=\frac{\operatorname{Cof}(0, j)}{\operatorname{det}(\mu-A)} \underset{N \rightarrow \infty}{\sim} \begin{cases}\frac{[\lambda+\varepsilon](-1)^{-j} x^{j+1}}{(x+\varepsilon-1)^{2}-(\lambda+\varepsilon)^{2}} & \text { if } j<0 \\ \frac{[x+\varepsilon-1](-1)^{j} x^{-j}}{(x+\varepsilon-1)^{2}-(\lambda+\varepsilon)^{2}} & \text { if } j \geqslant 0\end{cases}
$$

where

$$
x=\frac{\mu+2 \pm \sqrt{\mu^{2}+4 \mu}}{2}( \pm \text { such that }|x| \text { be maximum })
$$

After cumbersome computations, we get, for $N \rightarrow \infty$ :

$$
\begin{aligned}
\chi_{M_{0}}(\mu) & =G_{+}(\mu) G_{-}(\mu) \frac{\left(x_{+} x_{-}\right)^{2 N}}{\mu^{4}-16 \mu^{2}} \\
G_{+}(\mu) & =\left(x_{+}-1+2 \varepsilon+\lambda\right)\left(x_{-}-1-\lambda\right)+\lambda\left[\lambda+2 \varepsilon e^{2 \alpha}+2 e^{2 \alpha} \frac{x_{-}-x_{+}}{x_{+} x_{-}-1}\right] \\
G_{-}(\mu) & =\left(x_{-}-1+2 \varepsilon+\lambda\right)\left(x_{+}-1-\lambda\right)+\lambda\left[\lambda+2 \varepsilon e^{2 \alpha}+2 e^{2 \alpha} \frac{x_{+}-x_{-}}{x_{+} x_{-}-1}\right] \\
x_{+} & =\frac{\mu+2+\sqrt{\mu^{2}+4 \mu}}{2} \text { for } \operatorname{Re}(\mu)>-2 \\
x_{-} & = \begin{cases}\frac{-\mu+2+\sqrt{\mu^{2}-4 \mu}}{2} & \text { for } \operatorname{Re}(\mu) \in[0,2] \\
\frac{-\mu+2-\sqrt{\mu^{2}-4 \mu}}{2} & \text { for } \operatorname{Re}(\mu)>2\end{cases}
\end{aligned}
$$

Finally, one has to combine this last result with (31). This is done via the formula

$$
g(\alpha)=\frac{1}{4 i \pi} \oint d \mu\left[\mu \frac{\chi_{M_{0}}^{\prime}(\mu)}{\chi_{M_{0}}(\mu)}\right]-2 N+1-\varepsilon-\lambda
$$

where the contour for the complex integration is a big (big enough to enclose all singularities of the meromorphic function) half-circle leant on the imaginary axis, with its belly in 
the $\operatorname{Re}(\mu)>0$ region, followed counterclockwise. The logarithmic derivative of $\chi_{M_{0}}$ yields different additive contributions from formula (38). Let us consider first the contribution $\left(x_{+} x_{-}\right)^{2 N}$. The integral

$$
I=\frac{2 N}{4 i \pi} \oint d \mu\left[\mu \frac{x_{+}^{\prime}}{x_{+}}+\mu \frac{x_{-}^{\prime}}{x_{-}}\right]
$$

can be exactly computed, as the analytical singularities in the right half plane of $x_{+}$and $x_{-}$are located only on the real segments $[-4,0]$ and $[0,4]$ respectively; as a result, we can compute this integral with a contour encircling $[0,4]$ and sticking to it. Yet some care must be taken however, for the actual expression of $x_{-}$is not uniform on the whole half plane, and notably on the real axis: whereas $x_{+}$is everywhere given on $\operatorname{Re}(\mu)>0$ by $\left[\mu+2+\sqrt{\mu^{2}+4 \mu}\right] / 2, x_{-}$is given by $\left[-\mu+2+\sqrt{\mu^{2}-4 \mu}\right] / 2$ for $\operatorname{Re}(\mu) \in[0,2]$ and by $\left[-\mu+2-\sqrt{\mu^{2}-4 \mu}\right] / 2$ for $\operatorname{Re}(\mu)>2$. With all these precautions we get the simple result $I=2 N$. This extensive term vanishes with the corresponding term coming from $\operatorname{Tr}(A)$, which is coherent with the injection properties being asymptotically independent of the size of the system (in our 1D model).

We can also compute easily the contribution of the term $\left(\mu^{4}-16 \mu^{2}\right)^{-1}$. The associated integral yields -2 , thence we can recast the expression for $g(\alpha)$ into

$$
g(\alpha)=\frac{1}{4 i \pi} \oint d \mu \mu\left[\frac{G_{+}^{\prime}(\mu)}{G_{+}(\mu)}+\frac{G_{-}^{\prime}(\mu)}{G_{-}(\mu)}\right]-1-\varepsilon-\lambda
$$

\subsection{The case $\alpha=0$}

It is possible to verify partially the correctness of the formula. Indeed, when $\alpha=0$, we know the value of $g(\alpha): g(0)=0$, that maximum eigenvalue being associated with the stationary distribution (encoded in the corresponding eigenvector). Thus, we must verify this expected result, which holds whatever the values of $\lambda$ and $\varepsilon$.

To do that, we rewrite the expressions for $G_{+}$and $G_{-}$in a more transparent form. Using the relations $x_{ \pm}^{2}-( \pm \mu+2) x_{ \pm}+1=0$, one can write

$$
G_{+}(\mu)=\left(x_{-}-1\right)\left[\left(x_{+}-1\right)(1+2 \lambda / \mu)+2 \varepsilon\right]+2 \lambda \frac{e^{2 \alpha}-1}{x_{+} x_{-}-1}\left[\varepsilon\left(x_{+} x_{-}-1\right)+x_{-}-x_{+}\right]
$$

$G_{-}$is easily obtained by switching $x_{+}$and $x_{-}$, and making $\mu \rightarrow-\mu$ :

$$
G_{-}(\mu)=\left(x_{+}-1\right)\left[\left(x_{-}-1\right)(1-2 \lambda / \mu)+2 \varepsilon\right]+2 \lambda \frac{e^{2 \alpha}-1}{x_{+} x_{-}-1}\left[\varepsilon\left(x_{+} x_{-}-1\right)+x_{+}-x_{-}\right]
$$

\subsection{1 $\varepsilon=0, \alpha=0$}

This case is the simplest, since $G_{ \pm}=\left(x_{-}-1\right)\left(x_{+}-1\right)(1 \pm 2 \lambda / \mu)$ and the logarithmic derivative yields six contributions. The two contributions coming from $\left(x_{+}-1\right)$ and the contribution coming from $(1+2 \lambda / \mu)$ yield zero, as no branch cut/poles are associated with these functions in the half plane $\operatorname{Re}(\mu)>0$. Thus, from (45),

$$
\begin{aligned}
g(0) & =\frac{1}{4 i \pi} \oint d \mu \mu\left(\frac{2 x_{-}^{\prime}}{x_{-}-1}+\frac{1}{\mu-2 \lambda}\right)-1-\lambda \\
& =\frac{1}{4 i \pi} \oint d \mu \mu \frac{2 x_{-}^{\prime}}{x_{-}-1}-1
\end{aligned}
$$


The contour of integration can be stuck to the branch cut $[0,4]$ (that is $[0 \rightarrow 4]-i 0$ followed by $[4 \rightarrow 0]+i 0$. From $0-i 0$ to $2-i 0, x_{-}(\mu=y-i 0)=\left[-\mu+2+\sqrt{\mu^{2}-4 \mu}\right] / 2=$ $\left[-y+2+i \sqrt{4 y-y^{2}}\right] / 2$; from $2-i 0$ to $4-i 0$, the expression for $x_{-}(\mu)$ is changed, but the limit for $\mu$ sticking below the branch cut is such that one has still $x_{-}(\mu=y-i 0)=$ $\left[-y+2+i \sqrt{4 y-y^{2}}\right] / 2$. The expressions above the branch cut are just the complex conjugates. As a result $x_{-}$covers counterclockwise the set of complexes of modulus 1 , from $z=1(\mu=0-i 0)$ to $z=-1(\mu=4-i 0)$ with positive imaginary parts, and from $z=-1(\mu=4+i 0)$ back to $z=1$ with negative imaginary parts. As a result, we get the expected value:

$$
g(0)=\frac{1}{2 i \pi} \oint_{|z|=1} d z \frac{2-z-z^{-1}}{z-1}-1=0
$$

\subsection{2 $\varepsilon=1, \alpha=0$}

The computation in that case is slightly different, in particular as regards the localization of the singularities. The terms $\left(x_{+}-1\right)$ and $\left(x_{+}+1+2 \lambda \mu^{-1}\left(x_{+}-1\right)\right)$ have no singularities in the half plane under consideration (the singularity $\mu=0$ is avoided). The term $\left(x_{-}-1\right)$ yields a factor $1 / 2$, as already computed. The term

$$
x_{-}+1-2 \lambda \mu^{-1}\left(x_{-}-1\right)=-\frac{x_{-}-1}{x_{-}}\left(x_{-}^{2}+2 \lambda x_{-}-1\right)
$$

is the richest, as it has not only a branch cut, but also displays an isolated pole for $\mu=2\left[1+\sqrt{\lambda^{2}+1}\right]$. Thus,

$$
\begin{aligned}
g(0) & =-\frac{1}{4 i \pi} \oint d \mu \mu \frac{x_{-}^{\prime}}{x_{-}}+\frac{1}{2 i \pi} \oint d \mu \mu \frac{x_{-}^{\prime}}{x_{-}-1}-\frac{1}{2 i \pi} \oint_{|z|=1} \frac{d z}{z} \frac{(z-1)^{2}(z+\lambda)}{z^{2}+2 \lambda z-1}-1+\sqrt{\lambda^{2}+1}-\lambda \\
& =-\frac{1}{2 i \pi} \oint_{|z|=1} \frac{d z}{z} \frac{(z-1)^{2}(z+\lambda)}{z^{2}+2 \lambda z-1}-1+\sqrt{\lambda^{2}+1}-\lambda=0
\end{aligned}
$$

\subsection{The general case $(\alpha \neq 0)$}

The preceding particular cases show clearly that the analysis of the singularities becomes very complicated in the general case, as the new term proportional to $e^{2 \alpha}-1$ mixes $x_{+}$ and $x_{-}$. It is thus more appropriate to switch to an integral representation of the result. To achieve this in a simple way, we make use of the preceding verifications. We define

$$
I_{+}(\mu)=1+\frac{2 \lambda\left(e^{2 \alpha}-1\right)}{x_{-} x_{+}+1} \times \frac{1+\varepsilon\left(x_{-} x_{+}-1\right) /\left(x_{-}-x_{+}\right)}{\mu / 2+\lambda+\varepsilon \mu /\left(x_{+}-1\right)}
$$

and similarly $I_{-}$using the rules $x_{-} \leftrightarrow x_{+}$and $\mu \leftrightarrow-\mu$. Using the identity

$$
\frac{\left(x_{-}-x_{+}\right)\left(x_{-} x_{+}+1\right)}{\left(x_{+}-1\right)\left(x_{-}-1\right)\left(x_{-} x_{+}-1\right)}=2 / \mu
$$

one shows that

$$
g(\alpha)=\frac{1}{4 i \pi} \oint d \mu \quad \mu\left[\frac{I_{+}^{\prime}}{I_{+}}+\frac{I_{-}^{\prime}}{I_{-}}\right]
$$




\subsubsection{Model I $(\varepsilon=0)$}

The simplest expression is provided by the system $\mathrm{I}(\varepsilon=0)$ since in that case:

$$
I_{ \pm}=1+\frac{2 \lambda\left(e^{2 \alpha}-1\right)}{x_{-} x_{+}+1} \times \frac{1}{ \pm \mu / 2+\lambda}
$$

The integrand of (56) is of order $\mu^{-3}$, so the half circle of integration can be made infinite, the only non vanishing contribution being that of the vertical axis $\operatorname{Re}(\mu)=0$. It is easy to verify that on this axis $x_{+}=x_{-}^{*}$ and $x_{-}(-i y)=x_{-}(i y)^{*}$. As a result, one has

$$
\begin{aligned}
g(\alpha) & =\frac{1}{4 \pi} \int_{\infty}^{-\infty} d y y\left[\frac{1}{I_{+}} \frac{d I_{+}}{d y}+\frac{1}{I_{-}} \frac{d I_{-}}{d y}\right] \\
& =\frac{1}{4 \pi} \int_{-\infty}^{\infty} d y \log \left(\left|I_{+}\right|^{2}\right)=\frac{1}{2 \pi} \int_{0}^{\infty} d y \log \left(\left|I_{+}\right|^{2}\right)
\end{aligned}
$$

The explicit expression for $\left|I_{+}\right|^{2}$ is:

$$
\left|I_{+}(i y)\right|^{2}=1+\frac{4 \lambda^{2}\left(e^{2 \alpha}-1\right)\left(x_{-} x_{+}+e^{2 \alpha}\right)}{\left(x_{-} x_{+}+1\right)^{2}\left(\lambda^{2}+y^{2} / 4\right)}
$$

and

$$
\begin{aligned}
x_{-} x_{+} & =\left|x_{+}\right|^{2}=\frac{1}{4}\left|i y+2+\sqrt{-y^{2}+4 i y}\right|^{2} \\
& =\frac{1}{y}\left[\left(2+\frac{\sqrt{y}}{\sqrt{2}} \sqrt{-y+\sqrt{y^{2}+16}}\right)^{2}+\left(y+\frac{\sqrt{y}}{\sqrt{2}} \sqrt{y+\sqrt{y^{2}+16}}\right)^{2}\right]
\end{aligned}
$$

To summarize, we can write the result as

$$
\begin{aligned}
& g(\alpha)=\frac{2}{\pi} \int_{0}^{\infty} d u \log \left(1+\frac{\lambda^{2}\left(e^{2 \alpha}-1\right)\left(\psi(u)+e^{2 \alpha}\right)}{(\psi(u)+1)^{2}\left(\lambda^{2} / 4+u^{2}\right)}\right) \\
& \psi(u)=\left(1+\sqrt{2} \sqrt{-u^{2}+\sqrt{u^{4}+u^{2}}}\right)^{2}+\left(2 u+\sqrt{2} \sqrt{u^{2}+\sqrt{u^{4}+u^{2}}}\right)^{2}
\end{aligned}
$$

A useful relation for $\psi(u)$ can be deduced: from

$$
\frac{1}{x_{-} x_{+}+1}=\frac{1+x_{+} / x_{-}}{\left(1+x_{-} x_{+}\right)\left(1+x_{+} / x_{-}\right)}=\frac{1}{4}\left[\frac{1}{x_{+}}+\frac{1}{x_{-}}\right]
$$

one gets

$$
\frac{1}{\psi(u)+1}=\frac{1}{2}-\frac{1}{\sqrt{2}} \sqrt{-u^{2}+\sqrt{u^{4}+u^{2}}}
$$

This suggests the change of variables $u=1 / \sinh \tau$. This change gives the alternate expression:

$$
g(\alpha)=\frac{2}{\pi} \int_{0}^{\infty} d \tau \frac{\cosh \tau}{\sinh ^{2} \tau} \log \left(1+\frac{\lambda^{2}\left(e^{2 \alpha}-1\right)\left(\operatorname{coth}^{2} \frac{\tau}{4}+e^{2 \alpha}\right)}{\left(\operatorname{coth}^{2} \frac{\tau}{4}+1\right)^{2}\left(\frac{\lambda^{2}}{4} \sinh ^{2} \tau+1\right)} \sinh ^{2} \tau\right)
$$


A partial verification of the result can be done, via the computation of $g^{\prime}(0)$. This quantity equals the mean injected power in the stationary state, which can be computed directly by other means [20]. We have readily from (63) :

$$
\begin{aligned}
g^{\prime}(0) & =\frac{4 \lambda^{2}}{\pi} \int_{0}^{\infty} \frac{d u}{(\psi(u)+1)\left(\lambda^{2} / 4+u^{2}\right)} \\
& =2 \lambda\left(1+\lambda-\sqrt{\lambda^{2}+2 \lambda}\right)
\end{aligned}
$$

which is the expected result. Note that it is not surprising to find exactly the same result as in [20] despite the fact that here the system is duplicated with respect to the one studied in [20]. There, the energy associated with a domain wall was twice the value adopted here (1 per domain wall).

\subsubsection{Model II $(\varepsilon=1)$}

This model yields an expression slightly more complicated for $g(\alpha)$, due to an involved expression for $I_{+}$(formula (54) $)$. Some algebraic manipulations allow only a partial simplification :

$$
I_{+}(\mu)=1+\frac{2 \lambda\left(e^{2 \alpha}-1\right)}{\mu \psi(\psi+1)} \times \frac{2 \mu \psi-(\psi-1)^{2}}{\sqrt{\mu^{2}+4 \mu}+2 \lambda}
$$

where $\psi=x_{+} x_{-}$. This yields the result

$$
g(\alpha)=\frac{2}{\pi} \int_{0}^{\infty} d u \log \left|1+\frac{\lambda\left(e^{2 \alpha}-1\right)}{\psi+1} \times \frac{1+i(\psi-1)^{2} / 8 u \psi}{\lambda / 2+\sqrt{-u^{2}+i u}}\right|^{2}
$$

with $\psi=\psi(u)$ given by (64). This seemingly irreducible complexity we could have expected, since in contrast with model I, the mean injected power is here not computable by elementary operations (i.e. the dynamical equations for the correlators $\left\langle\sigma_{0} \sigma_{i}\right\rangle$ are not closed).

\section{The large deviation functions}

In this section, we discuss the properties of the ldf $f(p)$ associated with the integrated injected energy :

$$
\begin{aligned}
\operatorname{Prob}(\Pi / t=p) \underset{t \rightarrow \infty}{\propto} \exp (t f(p)) \\
f(p)=\min _{\alpha}(g(\alpha)-\alpha p)
\end{aligned}
$$

The typical shapes of the large deviation functions are plotted on figure 1, Their abscissae are rescaled to align their maximum on 1. The missing information on $\langle p\rangle$ is plotted on figure 2 for both models (obtained via $\langle p\rangle=g^{\prime}(\alpha=0)$ ). The mean injected power behaves monotonously in both models (fig. 21), with but a maximal asymptotic value twice larger (2) for model II. We verified the consistency of this result by a direct simulation of the model II (results not shown; for model I, we have the exact formula $\langle p\rangle=2 \lambda\left(\lambda+1-\sqrt{\lambda^{2}+2 \lambda}\right)$. That model II induces an injection larger than model I is obvious: the spin $s_{0}$ can switch back spontaneously and swiftly just after the creation of two domain walls, putting the system back in a state ready to accept anew a positive 

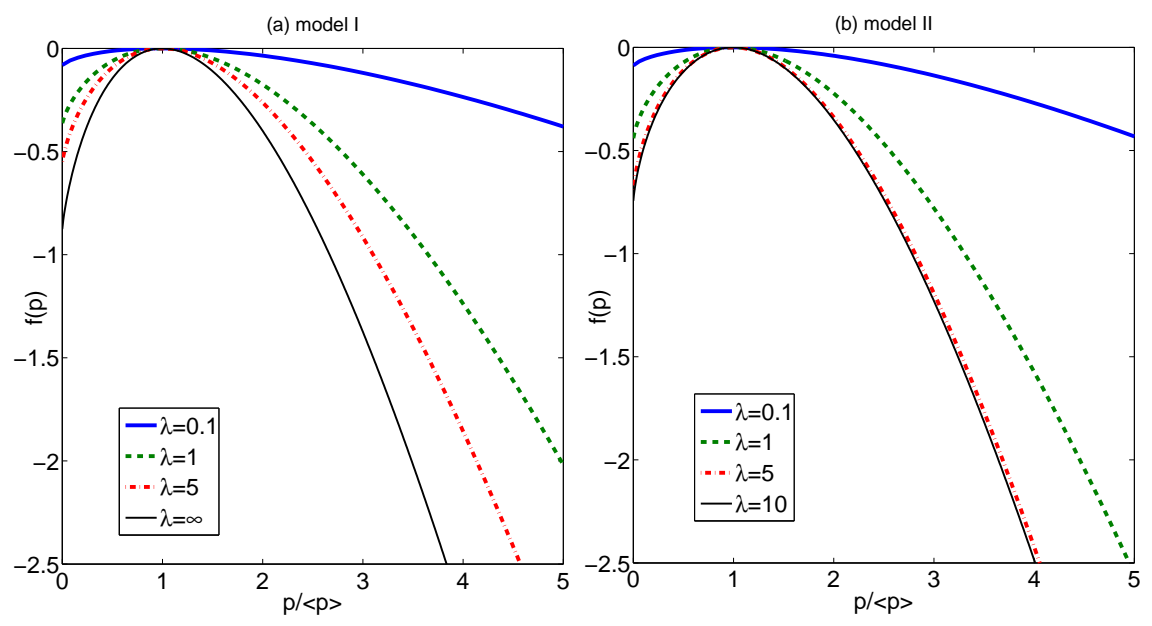

Figure 1: Typical shapes of the large deviation functions of model I (left) and II (right), for various values of $\lambda$. The abscissae only are rescaled. For model II, $\lambda=\infty$ is not plotted due to the particular analytical behaviour of $f$ in that case (see text); however we believe $\lambda=10$ to be close to the asymptotic case.

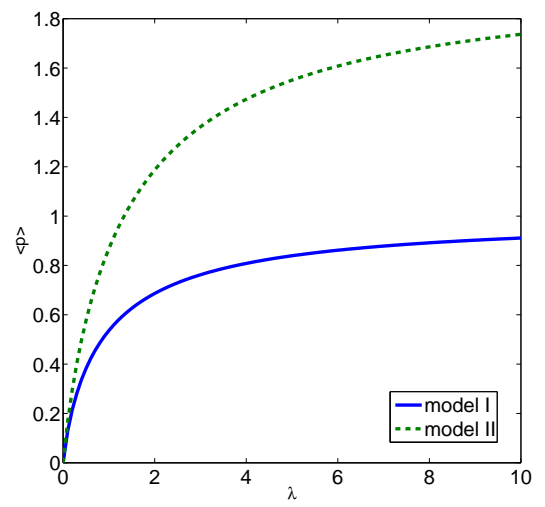

Figure 2: Mean injected power as a function of $\lambda$ for the two models considered.

injection of energy; in model I, the domain walls have to move away diffusively before such a state can be reached. It is interesting to note that for model II $\lim _{\lambda \rightarrow \infty} g^{\prime}(0)$ is not given by the simple limit taken inside the integral (which would have given $\langle p\rangle=1$ ). We postpone the discussion on this peculiarity to the end of the section.

The ldfs for both models have the interesting property that they have a finite limit when $p \rightarrow 0$ : arbitrary small integrated injected energies "are not so rare"; this feature would a priori favour the presence of a negative tail in the case where one averages over different initial conditions (as described in [9]); whether it is or not the case here is an issue beyond the scope of this paper.

The ldfs are apparently close to a parabola. This is of course not true, and the deviation from the parabola is a rather important feature to look at. To this end, one defines $\sigma(\lambda)=\left|f^{\prime \prime}(\langle p\rangle)\right| \times\langle p\rangle^{2}$, which inverse is a measure of the relative fluctuations of $\Pi$ (up to a $1 / \sqrt{\tau}$ factor). We see from figure 3 that $\sigma(\lambda)$ increases gently with $\lambda$ and reaches a constant value. This is consistent with the shrinking of $f$ with increasing $\lambda$ to be seen 


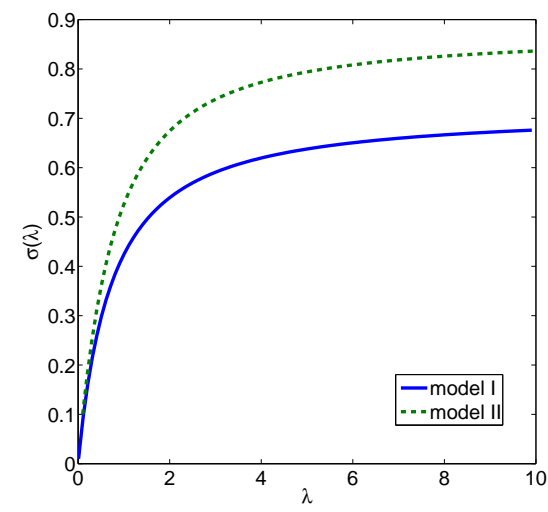

Figure 3: Parameter $\sigma(\lambda)=\left|f^{\prime \prime}(\langle p\rangle)\right| \times\langle p\rangle^{2}$ as a function of $\lambda$.

on figure 1 .

We then use $\sigma(\lambda)$ to rescale the ldfs so that the curvatures are equal to 1 , namely consider $f(p) / \sigma(\lambda)$ as a function of $p /\langle p\rangle$. The results are plotted for model I on figure 4. together with the reference parabola $-\frac{1}{2}(p /\langle p\rangle-1)^{2}$. Corresponding curves for model II are very similar, as can be seen on figure 5, where the ldfs for both systems with $\lambda=2$ (maximum discrepancy of parameters $\chi$, see later) are simultaneously plotted.

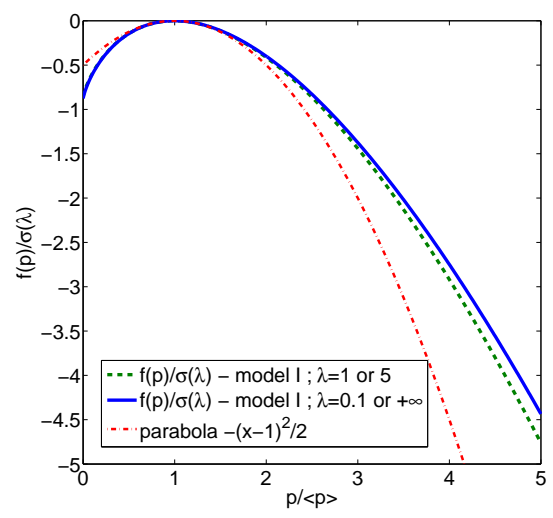

Figure 4: Rescaled large deviation functions for the model I. Note that the curves for $\lambda=0.1$ and $\lambda=\infty$ are almost identical, and this also true for $\lambda=1$ and $\lambda=5$. This is related to the non-monotonous behaviour of the parameter $\chi(\lambda)$, see text.

We remark that the ldf are rather different from a parabola, and display a marked tilt counterclockwise. Interestingly enough, the magnitude of the tilt is not a monotonous function of $\lambda$. This can be simply caught by inspecting $\chi(\lambda)=g^{\prime \prime \prime}(0) g^{\prime}(0) / g^{\prime \prime 2}(0)$, the third coefficient of the Taylor expansion of the scaled ldf near the maximum: $f(p) / \sigma(\lambda)+$ $\frac{1}{2}(p /\langle p\rangle-1)^{2} \simeq \frac{1}{6} \chi(p /\langle p\rangle-1)^{3}$. As shown on figure 6, $\chi(\lambda)$ has a non-monotonous behaviour with respect to $\lambda$, and mainly dictates the location of the asymptotic tail. Note that this parameter is positive, in accordance with the counterclockwise tilt already mentioned. The physical origin of these features is rather complicated. The tilt is mainly associated to the temporal correlations of the process, which are revealed in the statistics of rare events only. The probability of an extra injection of energy is more likely than the 


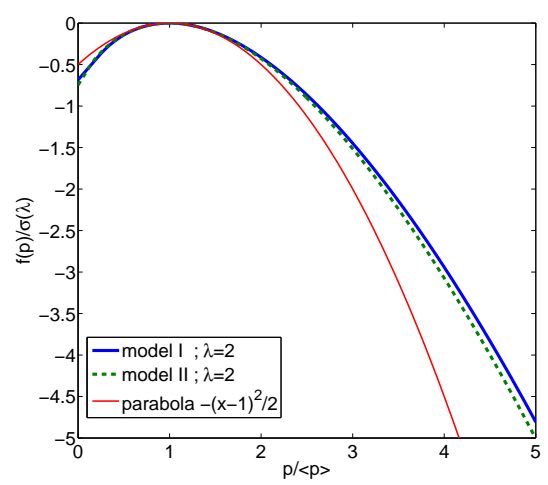

Figure 5: Rescaled large deviation functions for $\lambda=2$ in both models.

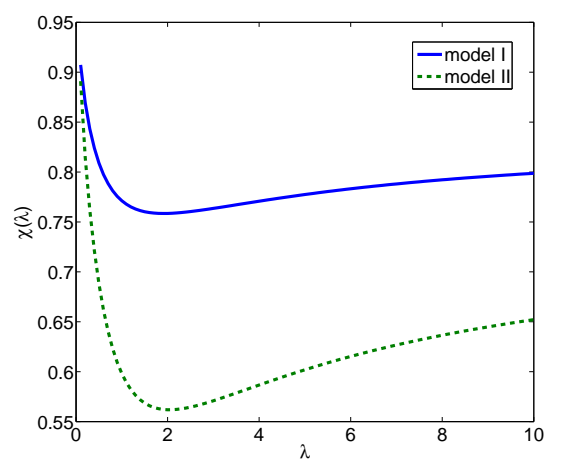

Figure 6: Asymmetry parameter $\chi(\lambda)$. See text for details

opposite, because of a kind of positive feedback : if for a while, an extra amount of energy has been already transfered into the system, the typical density of domain walls near the boundary experiences a positive fluctuation; as a result, subsequent incoming domain walls are more likely to eventually annihilate with an alter ego, instead of returning to the boundary (conversely a negative fluctuation is hard to maintain due to a growing shortage of domain walls to react with, see [9] for similar arguments).

That this positive feedback has a minimum is related to the structure of the stationary state. On figure 7 is plotted the repartition function of the domain wall nearest to $s_{0}$ in the stationary state for model I (we restrict the discussion to model I for sake of simplicity) and various values of $\lambda$ - these pdfs were obtained numerically although the exact computation is a priori tractable. It is seen that the probability for the first domain wall to be between spin 1 and spin 2 (that is, next to the first possible domain wall, which so to speak does not belong to the bulk as it is directly affected by the boundary flips) is maximum for $\lambda \sim 1-2$ (and is maximum with respect to the subsequent locations). This property is also true if one disregards the domain walls located in contact with the spin $s_{0}$ and restricts oneself to "inner" domain walls (see inset of fig [7). The correspondence between $\chi(\lambda)$ and the rescaled height of the peak 1-2 can be made quantitative by a correlation plot (fig. 7 (b)), where a linear relation between the two quantities can be approximately drawn.

Thus, a density fluctuation is typically localized very near the boundary especially for $\lambda \sim 2$, and this proximity works against the correlations and the positive feedback 
(a)

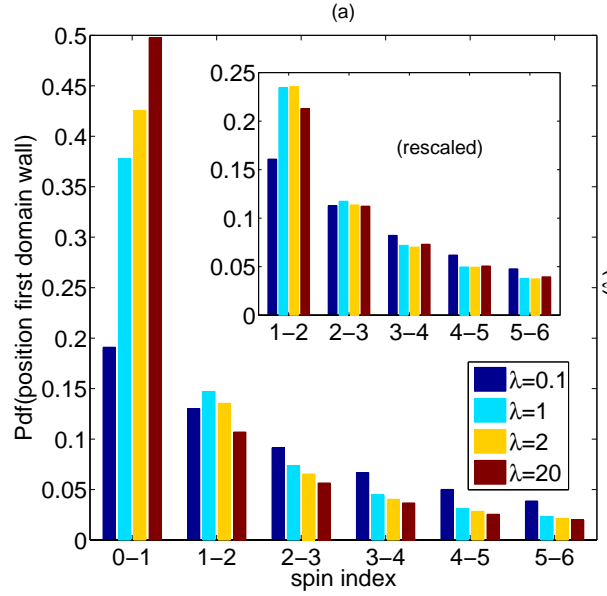

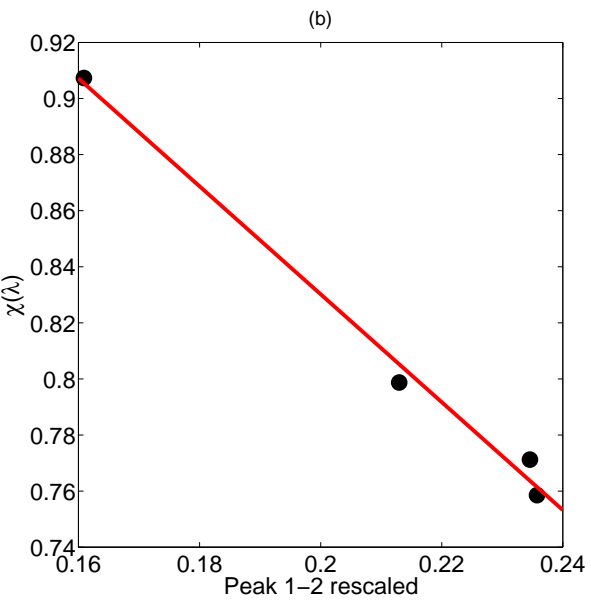

Figure 7: (a) Repartition function of the domain wall nearest to the spin 0 for model I. The inset shows the distribution function restricted (and rescaled in accordance) to the bulk domain walls. (b) Correlation plot between the height of the first peak (position 1-2) of the rescaled repartition function and $\chi(\lambda)$. The line is a linear fit.

effect explained above, as after just one move these domain walls can disappear through a negative energy injection event; this explains roughly the minimum of $\chi(\lambda)$ near $\lambda \sim 2$. Finally one explains also the less pronounced effect for model II by the fact that the two halves of the system see each other in that case and domain walls from one side can pervade the other: this permeability probably reduces the correlations between density fluctuations and injection, thereby decreasing the asymmetry parameter.

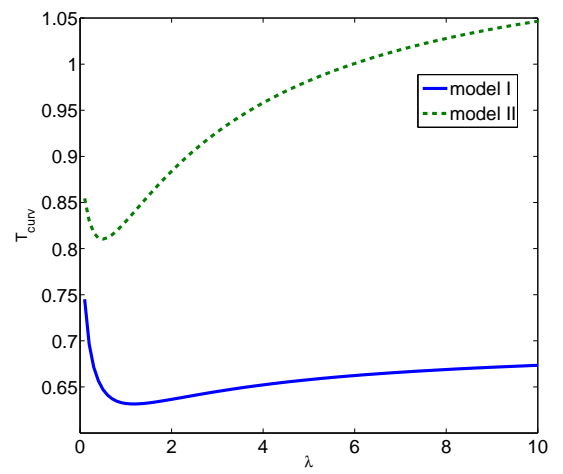

Figure 8: Nonequilibrium temperature $T_{\text {curv }}(\lambda)=g^{\prime \prime}(0) / 2 g^{\prime}(0)$. See text for details.

Interestingly enough, this parameter $\chi$ has a similar behaviour as the nonequilibrium "temperature" one can define for such dissipative NESS from $f(p)$ [10, 11] : $T_{\text {curv }}=$ $g^{\prime \prime}(0) / 2 g^{\prime}(0)$. Figure 8 shows this quantity for both models, and it is clear that this characteristic energy constructed from the fluctuations of $\Pi$ is sensitive to both the temporal correlations of the process (as $\chi$ ) and the averages quantities like the mean injected power. As such, it bears a rather complicated physical content, in contrast with an ordinary temperature concept.

Before to conclude, it is worth mentioning a mathematical subtlety of model II: the 
naive $\lambda \rightarrow \infty$ limit yields

$$
g(\alpha) \simeq \frac{2}{\pi} \int_{0}^{\infty} d u \log \left|1+\frac{2\left(e^{2 \alpha}-1\right)\left(1+i(\psi-1)^{2} / 8 u \psi\right)}{\psi+1}\right|^{2}
$$

whence one would have

$$
\begin{aligned}
g^{\prime}(0)=\langle p\rangle & =\frac{16}{\pi} \operatorname{Re}\left(\int_{0}^{\infty} d u \frac{1+i(\psi-1)^{2} / 8 u \psi}{\psi+1}\right) \\
& =1
\end{aligned}
$$

But this is not the correct result $(\langle p\rangle=2)$, and it is easy to see why : the exact formula for finite $\lambda$ is, provided $\sqrt{-u^{2}+i u}=R(u)+i I(u)$ :

$$
\begin{aligned}
& g^{\prime}(0)=\frac{8 \lambda}{\pi} \int_{0}^{\infty} d u \frac{\lambda / 2+R+I(\psi-1)^{2} / 8 u \psi}{(\psi+1)\left([\lambda / 2+R]^{2}+I^{2}\right)} \\
& R(u)=\frac{1}{\sqrt{2}} \sqrt{-u^{2}+\sqrt{u^{4}+u^{2}}} \\
& I(u)=\frac{1}{\sqrt{2}} \sqrt{u^{2}+\sqrt{u^{4}+u^{2}}}
\end{aligned}
$$

The missing part comes from the third term of the numerator : $I \sim u$ for large $u$, hence the associated integral is of order $1 / \lambda$ and not $1 / \lambda^{2}$ as naively expected. This problem is ultimately due to the fact that an isolated pole exists for model II at the right hand side of equation (56) (see section 21), an analytical property extremely sensitive to the form of the approximants: some sub-dominants terms are of primarily importance to keep the analytical structure untouched and makes allowance for the asymptotic locations of the singularities.

\section{Conclusion}

In this paper, we were able to compute exactly by fermionic techniques large deviation functions of the injected power for two 1D models of classical spins driven by a $T=$ 0 Glauber dynamics in the bulk and a Poissonian flip on the boundary. The results highlight the influence of the flipping rate on the low-frequency fluctuation properties of the injection; we showed in particular that the third derivative of $f$ is a sensible measure of the interplay between on one hand density fluctuations and on the other hand the ability for the external operator to transfer efficiently energy inside the system. By the way, we found and explained why this third derivative (or equivalently the third cumulant of the time-integrated injected energy) is always positive, a property surprisingly found also in conservative diffusive systems for the integrated current (see formula (4) in [17]); this similar behaviour is a bit surprising, as inner dynamics of dissipative and conservative systems are by nature much different.

\section{Acknowledgements}

We are much indebted to S. Aumaître, F. Cornu, S. Fauve and F. van Wijland for fruitful discussions. This work was supported by the ANR project JCJC-CHEF. 


\section{Appendix}

We give some details on the diagonalization of a Hamiltonian of the type:

$$
H=\sum_{n, m}\left[c_{n}^{\dagger} A_{n m} c_{m}+\frac{1}{2} c_{n}^{\dagger} B_{n m} c_{m}^{\dagger}+\frac{1}{2} c_{n} D_{n, m} c_{m}\right]
$$

where $A$ is symmetric real and $B$ and $D$ antisymmetric real. We postulate the transformation

$$
\begin{aligned}
c^{\dagger} & =V \xi^{\dagger}+U \xi \\
c & =U^{*} \xi^{\dagger}+V^{*} \xi
\end{aligned}
$$

where $U$ and $V$ are $2 N \times 2 N$ matrices. The fermionic structure is preserved if

$$
\begin{aligned}
& U U^{* T}+V V^{* T}=1 \\
& U V^{T}+V U^{T}=0
\end{aligned}
$$

It must be stressed that these equations are only $2 N(2 N+1)$ independent relations among $2(2 N)^{2}$ coefficients. Thus $2 N(2 N-1)$ relations can still be imposed to the coefficients of $U$ and $V$.

Next, we can replace (81) into (80) and extract four different contributions : a contribution made with terms $\xi \xi(N(2 N-1)$ terms $)$ and $\xi^{\dagger} \xi^{\dagger}(N(2 N-1)$ terms $)$ we want to get rid of :

$$
\begin{gathered}
\frac{1}{2}\left(\xi^{\dagger}\right)^{T}\left[V^{T} A U^{*}-U^{* T} A V+V^{T} B V+U^{* T} D U^{*}\right]\left(\boldsymbol{\xi}^{\dagger}\right) \\
\frac{1}{2}(\boldsymbol{\xi})^{T}\left[U^{T} A V^{*}-V^{* T} A U+U^{T} B U+V^{* T} D V^{*}\right](\boldsymbol{\xi})
\end{gathered}
$$

this gives precisely the $2 N(2 N-1)$ remaining relations on $U$ and $V$ (the matrices are antisymmetric); thirdly a contribution $\xi^{\dagger} \xi$

$$
\left(\boldsymbol{\xi}^{\dagger}\right)^{T} M(\boldsymbol{\xi}) \underset{\operatorname{def}}{=}\left(\boldsymbol{\xi}^{\dagger}\right)^{T}\left[V^{T} A V^{*}-U^{T} A U^{*}+\frac{1}{2}\left(V^{T} B U-U^{T} B V\right)+\frac{1}{2}\left(U^{* T} D V^{*}-V^{* T} D U^{*}\right)\right](\boldsymbol{\xi})
$$

Our goal is to diagonalize this matrix. If this is possible, the diagonalization is completed using $M=Q^{-1} \Delta_{M} Q$ and $\zeta=Q \xi, \zeta^{\dagger}=Q^{-1 T} \xi^{\dagger}$. But this is apparently not always possible, as the inspection of $M$ clearly shows. Two special situations allow however a diagonalization: if $B=-D, M$ is hermitian and therefore diagonalizable ; if $U$ and $V$ can be found real, $M$ is symmetric and also diagonalizable. But the last case we cannot immediately recognize. In the following we assume that a diagonalization of $M$ can be completed.

There is a fourth term in the decomposition, which is a constant arisen from the commutation $\xi \xi^{\dagger} \rightarrow \xi^{\dagger} \xi$. This constant reads

$$
\operatorname{Tr}\left[U^{T} A U^{*}+\frac{1}{2} U^{T} B V+\frac{1}{2} V^{* T} D U^{*}\right]=-\frac{1}{2} \operatorname{Tr}(M)+\frac{1}{2} \operatorname{Tr}(A)
$$

where relations among $U$ and $V$ were used. As a result, the Hamiltonian can be cast in the following form :

$$
H=\sum_{q} \Lambda_{q}\left(\xi_{q}^{\dagger} \xi_{q}-\frac{1}{2}\right)+\frac{1}{2} \operatorname{Tr}(A)
$$


where the $\Lambda_{q}$ are the eigenvalues of $M$. This formula differs slightly from those presented in [19] where the $\operatorname{Tr}(A)$ term is not written. It is interesting to see that the precise sign of $\Lambda_{q}$ is irrelevant, as far as the spectrum of $H$ is concerned. The eigenvalues of $H$ are actually given by

$$
\frac{1}{2}\left(\sum_{q} \Lambda_{q} \varepsilon_{q}+\operatorname{Tr}(A)\right)
$$

where the $\varepsilon_{q}$ are \pm 1 .

The preceding development essentially yields the conclusion embodied by the equation (89), provided that the diagonalization is possible. To study the diagonalization itself, it is particularly clever to follow the route described in [19], where in (81), the roles of $c$ and $\xi$ are reversed. Starting from (89) and using $\left[H, \xi_{q}\right]=\Lambda_{q} \xi_{q}$, one gets the following result: the spectrum of the matrix

$$
M_{0}=\left(\begin{array}{cc}
A & B \\
D & -A
\end{array}\right)
$$

is made with the eigenvalues of $M$. Actually it is exactly the spectrum of $M$ plus its symmetric with respect to zero : multiplying $\mu \mathrm{Id}-M_{0}$ by

$$
\left(\begin{array}{cc}
(\mu-A)^{-1} & 0 \\
(\mu+A)^{-1} D(\mu-A)^{-1} & (\mu+A)^{-1}
\end{array}\right)
$$

it is easily seen that the characteristic polynomial $\chi_{M_{0}}(\mu)=\operatorname{det}\left(\mu \mathrm{Id}-M_{0}\right)$ can be recast into

$$
\chi_{M_{0}}(\mu)=\chi_{A}(\mu) \chi_{A}(-\mu)(-1)^{\operatorname{dim}(A)} \operatorname{det}\left(1-B(\mu+A)^{-1} D(\mu-A)^{-1}\right)
$$

Next, using the relation $\operatorname{det}(1+A B)=\operatorname{det}(1+B A)$ valid whatever $A$ and $B$, and $\operatorname{det}(A)=$ $\operatorname{det}\left(A^{T}\right)$, it is readily seen that $\chi_{M_{0}}(\mu)=\chi_{M_{0}}(-\mu)$. Thus, the spectrum of $H$ is just duplicated in $M_{0}$ according to $\operatorname{Sp}\left(M_{0}\right)=\{\operatorname{Sp}(H),-\operatorname{Sp}(H)\}$

The question of the diagonalization of $H$ is closely related to the diagonalization of $M_{0}$. The case $D=-B$ is explicit in this representation since in that case $M_{0}$ is symmetric and thus diagonalizable with real eigenvalues. Actually, as the non diagonalization of a matrix is an accident rather than the rule, we can consider these cases as exceptional.

\section{References}

[1] R. Labbé, J. F. Pinton, and S. Fauve, J. Phys. II France,6,1099 (1996).

[2] S. Aumaître, S. Fauve, and J. F. Pinton, Eur. Phys. J. B,16,563 (2000).

[3] D. J. Evans, E. G. D. Cohen, and G. P. Morris, Phys. Rev. Lett.,71,2041 (1993).

[4] G. Gallavotti and E. G. D. Cohen, Phys. Rev. Lett., 74, 2694 (1995).

[5] J.Kurchan, cond-mat/0511073.

[6] S. Ciliberto and C. Laroche, J. Phys. IV (France),8,Pr6-215 (1998).

[7] K. Feitosa and N. Menon, Phys. Rev. Lett.,92, 164301 (2004).

[8] S. Aumaître, S. Fauve, S. McNamara, and P. Poggi, Eur. Phys. J. B,19,449 (2001). 
[9] J.Farago, J.Stat.Phys.,107 781 (2002).

[10] J.Farago, Physica A,331 69 (2004).

[11] S. Aumaître, J. Farago, S. Fauve and S. Mc Namara, Eur. Phys. J. B,42, 255-261 (2004).

[12] Andrea Puglisi, Paolo Visco, Alain Barrat, Emmanuel Trizac and Frédéric van Wijland, Phys. Rev. Lett.,95, 110202 (2005).

[13] P. Visco, A. Puglisi, A. Barrat, E. Trizac and F. van Wijland ,Europhys. Lett., 72, 55 (2005).

[14] E. Bertin, J. Phys. A: Math. Gen., 39, 1539 (2006).

[15] Vivien Lecomte, Zoltan Racz, Frederic van Wijland, J.Stat.Mech, P02008 (2005).

[16] Cornu F., private communication.

[17] T.Bodineau and B.Derrida, Phys.Rev.Lett.,92, 180601 (2004).

[18] B. Derrida and J. L. Lebowitz,Phys. Rev. Lett.,80 209 (1998).

[19] Malte Henkel, Enzo Orlandini, Jaime Santos, Ann. of Phys., 259, 163 (1997) ; cond-mat/9610059

[20] J.Farago, J.Stat.Phys.,118 373 (2005). 